ARTIGO

Recebido em: 19/09/2017

Aceito em: $25 / 06 / 2018$

\title{
Lei de Lotka: um olhar sobre a produtividade dos autores na literatura brasileira de finanças
}

\author{
Lotka's Law: a look at the productivity of the authors in the \\ brazilian literature of finance
}

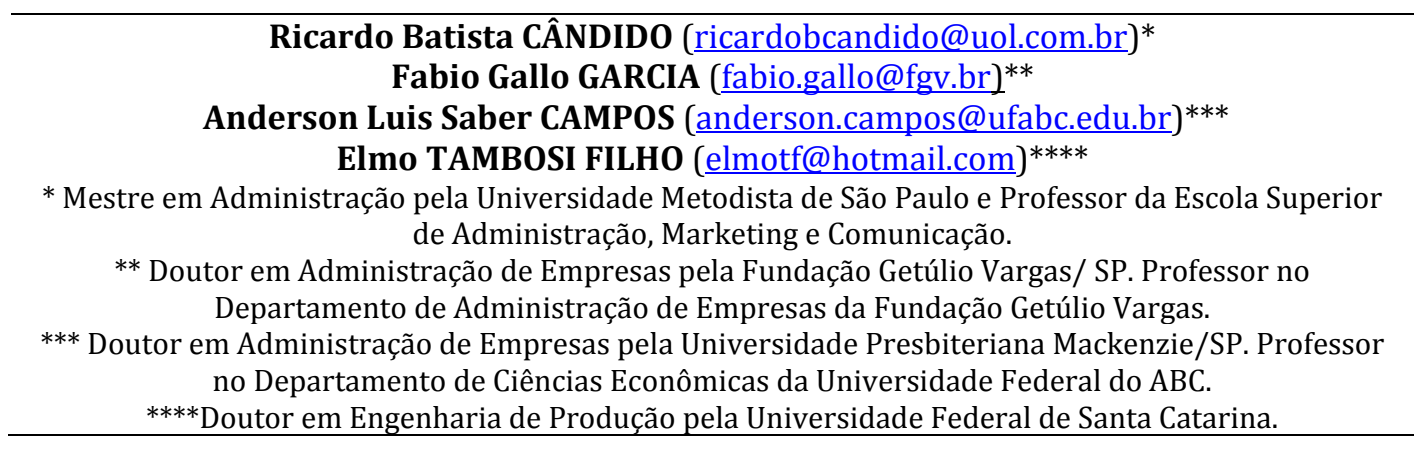

\section{Resumo}

No Brasil, as pesquisas em finanças tiveram início há cerca de 60 anos, entretanto, poucos estudos analisaram essa produção e, ao fazê-lo, se apropriaram devidamente das leis e princípios bibliométricos. A presente pesquisa investiga o nível de produtividade dos pesquisadores na literatura brasileira de Finanças aplicando a Lei de Lotka por contagem direta e completa. No corpus do estudo, composto por 873 artigos digitais, publicados em 24 conceituados periódicos nacionais entre 2005 e 2014 , verificou-se que a produtividade dos pesquisadores, quando feita pela contagem completa $(n=-$ $2,23)$, foi superior à contagem direta $(n=-2,89)$, sinalizando efeito favorável da produção sob regime de colaboração.

Palavras-chave: Finanças. Produtividade em pesquisa. Bibliometria. Lei de Lotka.

\begin{abstract}
In Brazil, research in finance began about 60 years ago, however, few studies analyzed this production and, in doing so, properly appropriated bibliometric laws and principles. The present research investigates the level of productivity of researchers in the Brazilian literature of Finance applying Lotka's Law by direct and complete counting. In the corpus of the study, composed of 873 digital articles, published in 24 well-known national journals between 2005 and 2014, it was verified that the productivity of the researchers, when done by the complete count $(n=-2.23)$, was superior to the direct count ( $n=-2.89$ ), indicating a favorable effect of production under a collaboration regime.
\end{abstract}

Keywords: Finance. Research productivity. Bibliometric. Lotka's Law.

v. 23, n. $53,2018$. p. $01-15$

ISSN 1518-2924 


\section{INTRODUÇÃO}

A área de Finanças, que em seus primórdios esteve associada às Ciências Contábeis e à Economia (HERLING; LIMA; MORITZ, 2013), a partir do século XIX assume um contorno mais administrativo (WESTON, 1977), passando a concentrar-se na busca da maximização de riqueza dos acionistas.

Desde então, acompanhando as demandas sociais de cada tempo, as pesquisas em finanças se expandiram passando por temas como estrutura e custo de capital, análise de risco, instrumentos de proteção financeira e avaliação de empresas, formando a base epistemológica que hoje a distingue das demais áreas do conhecimento (HERLING; LIMA; MORITZ, 2013).

Com o objetivo de compreender o comportamento produtivo dos pesquisadores da área de finanças, estudos bibliométricos têm sido realizados a exemplo dos trabalhos internacionais de Heck, Cooley e Hubbard (1986) que apontam a baixa produtividade dos pesquisadores de finanças norte-americanos e a dificuldade de se publicar mais de uma vez ao longo de 40 anos do Journal of Finance; o artigo de Cox e Chung (1990), que reafirma essa dificuldade e que discute a ocorrência do fenômeno "sucesso gera mais sucesso" na literatura de finanças; o trabalho nacional pioneiro de Leal, Oliveira e Soluri (2003) que contou com 551 artigos publicados entre 1974 e 2001, e que também expõe a baixa produtividade dos pesquisadores de finanças no Brasil; além trabalho de Camargos, Silva e Dias (2009), realizado sobre os Anais do Encontro da Associação Nacional dos Programas de PósGraduação em Administração (EnANPAD), que registra a baixa produtividade dos pesquisadores em função da grande frequência de autores com uma publicação, oriundos de Programas de Pós-Graduação Stricto Sensu .

Paralelamente ao crescimento dos estudos bibliométricos verificados no EnANPAD nos últimos anos (SPLITTER; DA ROSA; BORBA, 2011; TEIXEIRA; IWAMOTO; MEDEIROS, 2013), também têm crescido as críticas a esses trabalhos, em grande parte, por falhas metodológicas como: revisão bibliográfica insuficiente (MATTOS, 2004), dissociada dos estudos da Ciência da Informação (SPLITTER; DA ROSA; BORBA, 2011), ausência de justificativa na escolha de categorias conceito (MATTOS, 2004), e , em alguns casos, até mesmo, ausência total de indicadores bibliométricos (SPLITTER; DA ROSA; BORBA, 2011).

Propondo-se a contribuir com os já referenciados estudos bibliométricos nacionais da área de Finanças, a presente pesquisa recupera referências sobre o desenvolvimento da bibliometria e da Lei de Lotka; revisa estudos bibliométricos nacionais e internacionais aplicados à literatura de Finanças; amplia a base de periódicos pesquisados além de tratar detalhadamente do processo de análise da produtividade científica dos pesquisadores através da Lei de Lotka, seguindo uma metodologia validada pelo campo da Ciência da Informação cuja rotina é apresentada por Alvarado (2006).

0 corpus de estudo é formado por 873 artigos digitais, publicados nos principais periódicos nacionais da área de Administração, Ciências Contábeis e Turismo, que atualmente contam com classificação Qualis/CAPES ${ }^{1}$ A2, B1 e B2, no período compreendido entre 2005 e 2014.

Juntamente com a presente introdução, o trabalho contempla cinco partes organizadas sequencialmente. Na seção 2 apresenta-se a fundamentação teórica, abordando tanto o surgimento quanto o propósito da bibliometria além de resultados obtidos por estudos bibliométricos na área de Finanças; na seção 3 é apresentada a metodologia, amostra e dados; na seção 4 são apresentados e discutidos os resultados e, por fim, na seção 5 são apresentadas as principais contribuições do estudo juntamente com algumas sugestões para estudos futuros.

\section{REFERENCIAL TEÓRICO}

\footnotetext{
1 Qualis é o conjunto de procedimentos utilizados pela Capes para estratificação da qualidade da produção intelectual dos programas de pós-graduação. A classificação de periódicos é realizada pelas áreas de avaliação resultando no enquadramento dos veículos em estratos indicativos da qualidade, de A1 a C, sendo A1 o estrato mais elevado.
} 
Esta etapa, de revisão bibliográfica, apresenta um breve relato acerca do surgimento da bibliometria e dos estudos sobre produtividade dos autores, resgata estudos aplicados sobre a Lei de Lotka e conclui com a revisão de estudos, nacionais e internacionais, sobre produtividade dos autores na área de Finanças.

\subsection{Surgimento e propósito da bibliometria}

É no século XIX que surgem os primeiros estudos relacionados à área de estudo e serviço hoje denominada Ciência da Informação, tendo como pioneiro o belga Paul Outlet, que viveu entre 1868 e 1944 e que, segundo Mueller (2007), tinha como grande preocupação encontrar formas de controlar a informação visando garantir a possibilidade de acesso a quem dela precisasse.

Em meio ao movimento de consolidação da Ciência da Informação surge a bibliometria, técnica quantitativa e estatística de medição dos índices de produção e disseminação do conhecimento científico "[...] tal como procede a demografia ao recensear a população" (FONSECA, 1986, p.10), como uma resposta à necessidade de avaliação da atividade de produção e comunicação científica.

Araújo (2006) relata que abordagem semelhante à da bibliometria foi realizada, no início do século XX, sob a denominação de "bibliografia estatística", mas, aparentemente, sem grande adesão ao termo, o que é confirmado pela revisão feita por Guedes e Borschiver (2005) os quais constatam três utilizações do termo "bibliografia estatística" ao longo de quarenta anos.

Graças ao trabalho de Pritchard (1969) o termo bibliometria passa a designar o estudo de elementos objetivos da produção científica utilizando-se mais de métodos quantitativos do que discursivos comuns da bibliografia tradicional (ARAÚJO, 2006, p. 12).

\subsection{Lei de Lotka e a produtividade dos autores}

A primeira tentativa de se medir a produtividade dos autores de uma determinada área foi feita por Dresden (1922). Seu trabalho reúne 1.102 documentos, entre artigos e outras formas de comunicação escrita, publicadas no período compreendido entre 1879 e 1922, na Sociedade Americana de Matemática, seção Chicago, em que 278 autores contribuíram com a produção científica pesquisada.

Dresden (1922) constata para esse estudo um comportamento hiperbólico da distribuição, além de trazer detalhes acerca da dispersão e a concentração da distribuição de frequência da produtividade dos autores.

Após alguns anos, Lotka (1926) estuda a produtividade dos autores das áreas de Química e Física e identifica um comportamento padronizado da produtividade dos autores em diferentes áreas do conhecimento que será chamada, posteriormente, de Lei do Quadrado Inverso.

Segundo essa lei o número de autores que fazem " $n$ " contribuições em um determinado campo científico é aproximadamente $1 / \mathrm{n}^{2}$ daqueles que fazem uma só e que a proporção daqueles que fazem uma única contribuição é de mais ou menos $60 \%$.

Demonstração da importância e complexidade envolvida nos estudos sobre a produtividade científica em seus mais diversos campos é o fato de que, desde a apresentação do trabalho de Lotka (1926) até 2003, aproximadamente 390 trabalhos nas formas de livros, capítulos de livros, comunicações a congressos e literatura cinza, teriam sido publicados com o objetivo de criticar, replicar e/ou reformular esta lei (ALVARADO, 2007).

Para Alvarado (2007) uma das principais reformulações propostas à Lei de Lotka é substituição do Modelo do Quadrado Inverso pelo modelo do Poder Inverso Generalizado, proposto por Pao $(1985,1986)$ e Nicholls $(1986,1987,1989)$, que será aplicado neste trabalho.

\subsection{Estudos sobre produtividade de pesquisa em Finanças}

Um dos principais estudos que retrata a contribuição dos autores e instituições para a produção científica em Finanças é o de Heck, Cooley e Hubbard (1986), no qual os autores realizaram um levantamento sobre a publicação dos primeiros 40 anos (1946-1985) do Journal of Finance totalizando 2.182 artigos, dos quais participaram 1.788 autores. 
Sobre a contribuição individual dos 1.788 autores catalogados, a pesquisa demonstrou que, ao longo dos 40 anos, 1.202 (67,2\%) publicaram apenas uma vez, indicando baixa produtividade dos pesquisadores quando comparado com a padrões propostos por Lotka, de 60\%. Entretanto, quando comparadas a primeira década (19461945) e a última (1976-1985), o número de autores com apenas uma publicação diminuiu, caindo de $80 \%$ na primeira década para $67,4 \%$ na última, enquanto que o número de autores com cinco ou mais publicações aumenta, indo de $0,6 \%$ na primeira década para $5,5 \%$ na última, juntamente com o crescimento do número de coautorias (HECK; COOLEY; HUBBARD, 1986).

Seis anos depois, Heck e Cooley (2002) analisaram a contribuição dos autores mais produtivos em Finanças ao longo de cinquenta anos de publicação (1953-2002), contando com 72 periódicos, 29.717 artigos e notas catalogadas e 17.573 autores identificados.

Inicialmente, os autores agruparam 7 periódicos líderes em fator de impacto a partir de uma lista organizada por Borokhovich, Bricker e Simkins (1999), e verificaram que, 5.811 autores publicaram ao longo dos cinquenta anos, dos quais $3.302(56,8 \%)$ publicaram apenas uma vez; $932(16,1 \%)$ aparecem duas vezes; 466 (8,0 \%) aparecem três vezes; e 287 $(4,9 \%)$ aparecem quatro vezes.

No segundo agrupamento, com dezesseis periódicos, também organizado por fator de impacto, Heck e Cooley (2002) verificaram que, dentre todos os 11.475 autores que contribuíram para esse agrupamento $6.480(56,5 \%)$ aparecem apenas uma vez; 1.809 $(15,8 \%)$ aparecem duas vezes, $912(7,9 \%)$ aparecem três vezes; e $527(4,6 \%)$ aparecem quatro vezes, reproduzindo uma distribuição relativamente semelhante.

Entretanto, os autores chamam a atenção para o fato de que alguns líderes em publicações do primeiro ranking, com sete periódicos, perdem muitas posições quando considerados dezesseis periódicos e quando comparadas às formas de atribuição de pontos (direta, quando se atribui um ponto inteiro a cada coautor, e fracionada, quando os coautores de uma publicação dividem igualitariamente um ponto pela publicação).

Por fim, Heck e Cooley (2002) analisam os autores mais produtivos nas 72 revistas de Finanças relatando que dos 17.573 autores 10.275 (58,5\%) publicaram apenas uma vez; $2.573(14,6 \%)$ aparecem duas vezes; $1.317(7,5 \%)$ aparecem três vezes; 789 (4,5\%) aparecem quatro vezes, e que para ser classificado entre os 367 autores mais produtivos o autor deveria ter contribuído com, pelo menos, 18 artigos.

Os autores observam que a média de publicações, quando considerada apenas a última década do estudo, permaneceu constante colocando contrapondo a ideia de que a pressão por publicação nos últimos anos teria impactado decisivamente na produção; e que as coautorias teriam aumentado significativamente quando comparado a década de 60 , em que $6,7 \%$ dos artigos eram feitos em parceria, ao ano 2000, em que esse percentual aumentou para 58,3\% (HECK; COOLEY, 2002).

Outro estudo amplamente citado nas pesquisas bibliométricas é o de Cox e Chung (1990), intitulado Patterns of Produtivity in the Finance Literature: A Study of the Bibliometric Distribuition, no qual os autores fazem referência ao fenômeno das citações em que artigos, autores e periódicos frequentemente citados, referenciados e ou acessados terão privilégios frente aos demais congêneres menos conhecidos resultando no fenômeno conhecido como "sucesso gera mais sucesso".

Para esse estudo, os autores analisaram as publicações de 15 periódicos devotados à área de Finanças, no ano de 1986, e realizaram uma análise à luz do Modelo do Quadrado Inverso de Lotka, buscando testar sua aplicabilidade àquela amostra. Para testar a adequação da distribuição empírica e teórica, os autores utilizaram o teste $\chi^{2}$ - chi-quadrado - ao nível de 5\% de significância.

Incialmente o trabalho reconhece a dificuldade de se publicar mais de uma vez quando relata que dos 6.270 autores relacionados no estudo, a maioria dos autores $(62,2 \%)$ publicaram apenas uma vez e que apenas 15,4\% publicaram mais de 3 vezes.

Os autores concluem que, quando somado um grande número de revistas, os resultados da produtividade em Finanças se aproximam daquele apontado por Lotka (1926), calculado pelo Modelo do Quadrado Inverso, portanto, com expoente $n=2$, contudo, quando analisados os periódicos individualmente, os resultados dos expoentes variam de 1,95 a 3,26, 
com forte concentração de trabalhos dos pesquisadores mais produtivos nos periódicos de maior impacto, reforçando a tese da "riqueza que gera mais riqueza".

Posteriormente, Chung e Cox (1991) realizam novo estudo bibliométrico, agora testando a produtividade de pesquisa na literatura de Economia, utilizando como base de dados 20 periódicos, ao longo de um período de 26 anos. 0 objetivo do estudo foi de comprovar que artigos publicados em um pequeno número de periódicos contribuem para a maioria das referências na literatura de economia.

Foram feitos testes para o conjunto de periódicos e para cada periódico isoladamente, aplicando a Lei de Lotka, pelo Modelo do Poder Inverso Generalizado, testado ao nível de $1 \%$ de significância.

Seus resultados apontam para a grande dificuldade de se repetir publicações em economia, sendo que dos 13.576 autores identificados no estudo $57,7 \%$ publicaram apenas uma vez, contudo, alguns periódicos apontaram taxas de até $81,5 \%$ de autores de apenas uma publicação.

Segundo Chung e Cox (1991) os resultados dessa segunda pesquisa convergem com os do primeiro trabalho e, portanto, confirmam a capacidade preditora da Lei de Lotka quando aplicada a um conjunto de periódicos, apresentando $n=-1,84$, no entanto, não sendo tão eficiente quando aplicado aos periódicos isoladamente, com coeficientes " $n$ " que variam de 2,04 a 3,11 .

No Brasil, buscando identificar o perfil da pesquisa em Finanças, Leal, Oliveira e Soluri (2003) desenvolveram um estudo que contou com 551 artigos da área, publicados entre 1974 e 2001, obtidos em cinco periódicos nacionais de referência da área de Administração, além de 264 artigos incluídos nos anais do maior encontro brasileiro de pesquisa em administração, o Encontro da Associação Nacional dos Programas de PósGraduação em Administração (EnANPAD).

Os autores constataram que grande parte da produção se concentrava em reduzido número de pesquisadores, com $70 \%$ deles publicando apenas um artigo, e que o coeficiente generalizado foi de $n=-2,44$, denotando produtividade abaixo do padrão definido pela Lei de Lotka, além de haver forte concentração de publicações nas Instituições de Ensino Superior (IES) da região Sul e Sudeste.

Passados dez anos dessa primeira experiência de pesquisa sobre a produção brasileira em Finanças, Leal, Almeida e Bortolon (2013) realizaram novo estudo, com enfoque quali quantitativo, contando com 11 periódicos científicos nacionais, das áreas de Administração, Economia, Contabilidade e Produção, classificados à época no extrato Qualis/Capes A2, com o objetivo de analisar o comportamento dos pesquisadores em relação à coautoria, áreas temáticas e produtividade dos autores.

Utilizando o Modelo do Quadrado Inverso, os autores concluíram que a produtividade científica brasileira de Finanças ficou abaixo do previsto pela teoria, com $n=2,63$; e também da produtividade norte-americana como relatado no estudo de Cox e Chung (1990); que $72 \%$ dos autores publicaram apenas um artigo; e que os mais produtivos, que publicaram cinco artigos ou mais artigos, representaram apenas 5\% dos 643 autores identificados na amostra.

Segundo os autores, o elevado número de autores com apenas um artigo indica que grande parte dessas publicações deriva de dissertações de mestrado, programa que cada vez mais prepara para o mercado (VASCONCELOS et al., 2013).

Ampliando os estudos que tratam da produção científica nacional em Finanças, Camargos, Silva e Dias (2009), realizaram um levantamento nos anais do EnANPAD, agora dos artigos publicados entre 2000 e 2008, e que contou com 391 artigos distribuídos em seis subáreas temáticas que também foram subdivididas em 26 temas de pesquisa.

Nessa pesquisa, que aplicou o Modelo do Quadrado Inverso, o coeficiente estimado para a produtividade de pesquisa, de $n=2,37$, foi maior que o previsto pela Lei de Lotka (1926), demonstrando que o número de autores que publicam apenas um artigo está acima da prevista pela teoria bibliométrica, corroborando com os resultados de (LEAL; OLIVEIRA; SOLURI, 2003; VASCONCELOS et al., 2013).

Os autores concluem que, em parte, esse comportamento se deve ao rigor na seleção dos trabalhos, mas também à baixa efetividade das publicações em eventos, que muitas 
vezes só ocorrem pela exigência de publicação dos trabalhos de conclusão em programas de mestrado e doutorado (CAMARGOS; SILVA; DIAS, 2009).

\section{MATERIAL E MÉTODOS}

A presente pesquisa é descritiva exploratória (GIL, 2010; VERGARA, 2014) e utiliza fonte de dados de natureza primária haja vista o pesquisador atuar sobre esses dados desde a escolha da amostra até seu tratamento e análise (RICHARDSON, 2015).

Algumas estratégias iniciais de amostragem foram elaboras tomando como principal referência o extrato Qualis/CAPES, entretanto, se mostraram inadequadas, resultando em número restrito de periódicos, basicamente por três motivos: i) às inúmeras mudanças ocorridas nos critérios de classificação Qualis/CAPES ao longo de todo o período; ii) à instabilidade dos periódicos nos estratos e; iii) ao fato de muitos periódicos brasileiros ainda possuírem uma grande base de dados não digitalizada.

Optou-se, então, por uma amostra não probabilística, intencional ou de seleção racional (RICHARDSON, 2015), também conhecida como amostra por julgamento (COSTANETO, 1977), em que o pesquisador deliberadamente escolhe os elementos que comporão a amostra, amparando-se na sua experiência para definir os elementos mais representativos.

Foram selecionados, inicialmente, 53 reconhecidos periódicos nacionais, classificados no sistema Qualis/CAPES pela área de Administração, Ciências Contábeis e Turismo, nos extratos A2, B1 e B2 no ano de 2014, sendo que não foram encontrados periódicos nacionais no estrato A1 no referido relatório.

Filtrando-se por disponibilidade em formato digital e tempo de publicação, a amostra foi reduzida a 24 periódicos que juntos, ao longo dos dez anos de estudo, publicaram 7.541 artigos, distribuídos em 909 exemplares, sendo retirados da amostra editoriais, resenhas, reedições, traduções, participações especiais e pensatas, restando apenas as contribuições originais.

Da visita aos sites dos periódicos, ou ao site da Scielo (Scientific Electronic Library Online), foram baixados por download 1.050 artigos afetos à área de Finanças, todos em formato PDF, e que, posteriormente, foram convertidos em formato Word ${ }^{\circledR}$, sendo algumas informações transportadas para uma planilha Excel®.

Os metadados de descrição como Título, Resumo e Palavras-chave de cada artigo foram agrupados em apenas uma célula da planilha dando origem à variável titulo_resumo_key que, tratadas com auxílio do software para análise de dados qualitativos e quantitativos Sphinx Lexica ${ }^{\circledR}$ [http://www.sphinxbr.com.br], permitiu a identificação de três artigos duplicados, resultando em 1047 observações, sobre as quais aplicou-se a técnica de análise de conteúdo na sua forma clássica (RICHARDSON, 2015), com o propósito fundamental de identificar artigos que não seriam propriamente da área de Finanças, além de descrever aspectos quantitativos da produção.

Buscando reduzir os efeitos de um possível viés de amostra, o processo de retirada dos artigos classificados como de outras áreas foi composto por três fases: a primeira, de pré-classificação, feita por leitura da variável titulo_resumo_key; a segunda, composta pela leitura do texto na íntegra e, por fim, e em caso de dúvida, uma terceira fase de consulta de especialistas.

Após essa etapa foram encontrados e retirados 174 artigos relacionados às áreas de Contabilidade, Economia, Gestão Estratégica e Finanças Públicas, resultando na amostra final de $873(11,58 \%)$ artigos, distribuídos nos 24 periódicos, apresentados no Quadro 1. Os dados estão dispostos em ordem alfabética pelo nome do periódico; identificados por seus respectivos ISSNe, além de trazer seus acrônimos, suas classificações Qualis/CAPES no ano de 2014 e seus respectivos editores. 
Quadro 1: Identificação dos 24 periódicos que compuseram a amostra

\begin{tabular}{|c|c|c|c|c|}
\hline ISSNe & Nome do Periódico (Online) & Sigla & Estrato 2014 & Editor \\
\hline 1807-7692 & Brazilian Administration Review & BAR & A2 & ANPAD \\
\hline $1808-2386$ & Brazilian Business Review (English Edition. Online) & BBR & B1 & FUCAPE \\
\hline 1984-5146 & Brazilian Review of Finance & RBF & B2 & SBF \\
\hline $1679-3951$ & Cadernos EBAPE.BR & CEBAPE & A2 & FGV/EBAPE \\
\hline $1980-5330$ & Economia Aplicada & EA & B1 & FEARP \\
\hline $1982-3533$ & Economia e Sociedade & ES & B1 & UNICAMP/IE \\
\hline $1806-9649$ & Gestão \& Produção & GP & B1 & UFSCAR \\
\hline $1984-9230$ & Organizações \& Sociedade & osoc & A2 & UFBA/EA \\
\hline $1980-5411$ & Produção & PROD & B2 & ABEP \\
\hline 1806-9134 & Revista Brasileira de Economia & RBE & B1 & FGV/EPGE \\
\hline 1983-0807 & Revista Brasileira de Gestão de Negócios & RBGN & A2 & FECAP \\
\hline 1808-057X & Revista Contabilidade \& Finanças & RCF & A2 & USP/FIPECAF \\
\hline $1982-7849$ & Revista de Administração Contemporânea & RAC & $\mathrm{A} 2$ & ANPAD \\
\hline $1679-5350$ & Revista de Administração da Unimep & RAU & B2 & UNIMEP \\
\hline $1984-6142$ & Revista de Administração da USP & RAUSP & A2 & USP/FEA \\
\hline $2178-938 x$ & Revista de Administração de Empresas & RAE & A2 & FGV/EAESP \\
\hline $1676-5648$ & Revista de Administração de Empresas & RAE_E & A2 & FGV/EAESP \\
\hline $1809-2039$ & Revista de Administração e Inovação & RAI & B2 & USP \\
\hline $1678-6971$ & Revista de Administração Mackenzie & RAM & B1 & UPM \\
\hline $1982-3134$ & Revista de Administração Pública & RAP & A2 & FGV/EBAPE \\
\hline $2175-8077$ & Revista de Ciências da Administração & RCA & B2 & UFSC/DCA \\
\hline $1980-5527$ & Revista de Economia Contemporânea & REC & B1 & UFRJ/IE \\
\hline $1413-2311$ & Revista Eletrônica de Administração & READ & B1 & UFRGS/EA \\
\hline 1809-3337 & Revista Universo Contábil & RUC & B1 & FURB \\
\hline
\end{tabular}

Fonte: Elaborado pelos autores.

A produtividade dos pesquisadores foi calculada utilizando-se o modelo conhecido como Poder Inverso Generalizado, seguindo a rotina proposta por Alvarado (2006), cuja distribuição teórica é calculada pela equação (1), utilizando planilha eletrônica Excel®:

$$
\gamma_{x}=c x^{-n}
$$

Para $x=1,2,3, \ldots, x \_$max

Onde:

$\gamma_{x}=$ é a probabilidade de que um autor faça $x$ contribuições sobre um assunto;

$c$ e $n=$ são os dois parâmetros a serem estimados a partir dos dados observados.

O parâmetro $c$ que representa a porcentagem teórica dos autores que contribuíram com apenas um artigo ao longo do período estudado é calculado pela função Zeta Inversa de Riemann, ajustada por Pao (1985), conforme a equação (2). 


$$
c=\frac{1}{\sum_{x=1}^{P-1} \frac{1}{x^{n}}+\frac{1}{(n-1) P^{n-1}}+\frac{1}{2 P^{n}}+\frac{n}{24(P-1)^{n+1}}}
$$

Onde:

$x=$ é o número de $1,2,3, \ldots$ contribuiç̃oes por autor

$n=$ é o valor do parâmetro calculado pela equação (3).

$P=$ é o número de pares observados

0 parâmetro $n$, relativo à declividade da Lei de Lotka foi calculado por mínimos quadrados lineares utilizando a equação (3).

$$
n=\frac{N \sum X Y-\sum X \sum Y}{N \sum X^{2}-\left(\sum X\right)^{2}}
$$

Onde:

$N=$ número de pares de dados.

$X=\log \mathrm{x}$ na base 10.

$Y=$ Log de y na base 10 .

A contagem dos autores é desenvolvida por contagem direta, permitindo comparar os resultados dessa pesquisa com o padrão apresentado por Lotka (1926), além da contagem completa, a qual reflete com maior exatidão o comportamento produtivo contemporâneo.

A série de dados estudada é de 10 anos, buscando assim satisfazer às exigências da corrente defendida por Price (1963) e Potter (1981) e que já vem sendo seguido por outros estudos.

No teste de ajustamento das distribuições, empírica e teórica, foi aplicado o teste KS (Kolmogorov-Smirnov) ${ }^{2}$ ao nível de significância de $\alpha=0,01$, sendo que, a escolha pelo KS darse-á em decorrência da ineficiência do teste $\chi^{2}$ (chi-quadrado) quando aplicado à baixas frequências, o que nos obrigaria a combinar frequências de diferentes categorias, incorrendo em perda de informação (SIEGEL, 1975).

\section{APRESENTAÇÃO E DISCUSSÃO DOS RESULTADOS}

Nesta fase são apresentados e discutidos os resultados obtidos com a análise descritiva dos dados, fundamentalmente relacionados ao número de publicações, de autores e de colaborações além dos resultados obtidos com a aplicação da Lei de Lotka, calculada pelo modelo do Poder Inverso Generalizado, por contagem direta e contagem completa.

\subsection{Análise descritiva dos dados}

Ao longo do período estudado, a publicação científica de Finanças apresentou incremento de $54 \%$, o que representa uma taxa média linear de crescimento da ordem de $5,97 \%$ ao ano, contudo, tal crescimento não foi contínuo.

É possível verificar, com auxílio da Tabela 1, que entre 2005 e 2009 o número de publicações cresceu quase que continuamente, saltando de 62 para 108 artigos, portanto, $74 \%$ de crescimento no período, posteriormente, passou por uma redução em 2010, da ordem de - 27\%; retomou o crescimento em 2011 e 2012 recuperando o nível de produção de 2009, entretanto, sofreu uma nova redução em 2013 e 2014, também da ordem de -16\%.

Parece razoável sugerir que o movimento cíclico das publicações de Finanças no Brasil esteja relacionado com os ciclos de avaliação dos programas de Pós-Graduação Stricto

\footnotetext{
20 teste Kolmogorov-Smirnov é um teste não paramétrico sobre a igualdade de distribuições de probabilidade contínuas e unidimensionais. Recebe este nome em homenagem aos matemáticos russos Andrei Kolmogorov e Nikolai Smirnov.
} 
Sensu, antigamente trimestrais e hoje quadrienais, ocorridos em 2007, 2010 e 2013, e que costumam apresentar baixa produção nos anos iniciais dos ciclos compensados por maior produção nos anos finais.

Tabela 1: Produção científica em Finanças de 2005 a 2014

\begin{tabular}{lccccccccccc}
\hline & 2005 & 2006 & 2007 & 2008 & 2009 & 2010 & 2011 & 2012 & 2013 & 2014 & Totais \\
\hline Produção de Finanças & 62 & 74 & 69 & 91 & 108 & 78 & 97 & 108 & 96 & 90 & 873 \\
\% Crescimento & - & 19,35 & $-6,76$ & 31,88 & 18,68 & $-27,78$ & 24,36 & 11,34 & $-11,11$ & $-6,25$ & 53,72 \\
\hline
\end{tabular}

Fonte: Elaborado pelos autores.

Envolvidos na produção dos 873 artigos foram identificados 1.250 diferentes autores, portanto, uma média de 1,43 autores/artigo, entretanto, contabilizando autorias e coautorias encontramos 2.223 ocorrências (participações), produzindo uma média de 2,55 autores/ artigo.

A análise de autores/artigo, que é uma análise de input (insumos), permite antecipar a compreensão acerca da organização dos pesquisadores em torno das pesquisas. Assim, é possível verificar na Tabela 2 que o número de ocorrências de coautoria segue uma tendência de crescimento, interrompida apenas pelos anos de queda das publicações, o que reforça os resultados encontrados por (CAMARGOS; COUTINHO; AMARAL, 2005; HECK; COOLEY, 2002; VASCONCELOS et al., 2013) sobre o aumento do número de parcerias em pesquisas nas publicações nacionais nos últimos anos.

Tabela 2: Evolução da produção, autores e parcerias de 2005 a 2014

\begin{tabular}{cccccc}
\hline Ano & Autores & Coautoria & Produção & $\begin{array}{c}\text { Autor por } \\
\text { artigo }\end{array}$ & $\begin{array}{c}\text { Coautoria por } \\
\text { artigo }\end{array}$ \\
\hline $\mathbf{2 0 0 5}$ & 111 & 133 & 62 & 1,79 & 2,15 \\
$\mathbf{2 0 0 6}$ & 122 & 172 & 74 & 1,65 & 2,32 \\
$\mathbf{2 0 0 7}$ & 94 & 164 & 69 & 1,36 & 2,38 \\
$\mathbf{2 0 0 8}$ & 113 & 205 & 91 & 1,24 & 2,25 \\
$\mathbf{2 0 0 9}$ & 160 & 280 & 108 & 1,48 & 2,59 \\
$\mathbf{2 0 1 0}$ & 116 & 206 & 78 & 1,49 & 2,64 \\
$\mathbf{2 0 1 1}$ & 131 & 274 & 97 & 1,35 & 2,82 \\
$\mathbf{2 0 1 2}$ & 155 & 289 & 108 & 1,44 & 2,68 \\
$\mathbf{2 0 1 3}$ & 126 & 255 & 96 & 1,31 & 2,66 \\
$\mathbf{2 0 1 4}$ & 122 & 245 & 90 & 1,36 & 2,72 \\
\hline Totais & $\mathbf{1 2 5 0}$ & $\mathbf{2 2 2 3}$ & $\mathbf{8 7 3}$ & $\mathbf{1 , 4 3}$ & $\mathbf{2 , 5 5}$ \\
\hline
\end{tabular}

Fonte: Elaborado pelos autores.

Analisando o número de autores por artigo numa perspectiva longitudinal, como pode ser visto na Figura 1, nota-se que a frequência de dois autores foi a que mais ocorreu por quase todos os anos, entretanto, a frequência de três autores parece estar se tornando a nova tendência de organização de coautorias, chegando a superar a frequência de artigos com dois autores em 2012 e 2014.

A frequência de quatro autores também cresceu de 2007 a 2012, contudo, apresentou redução nos últimos três anos, enquanto que as produções com um e cinco autores apresentaram comportamento estável. 


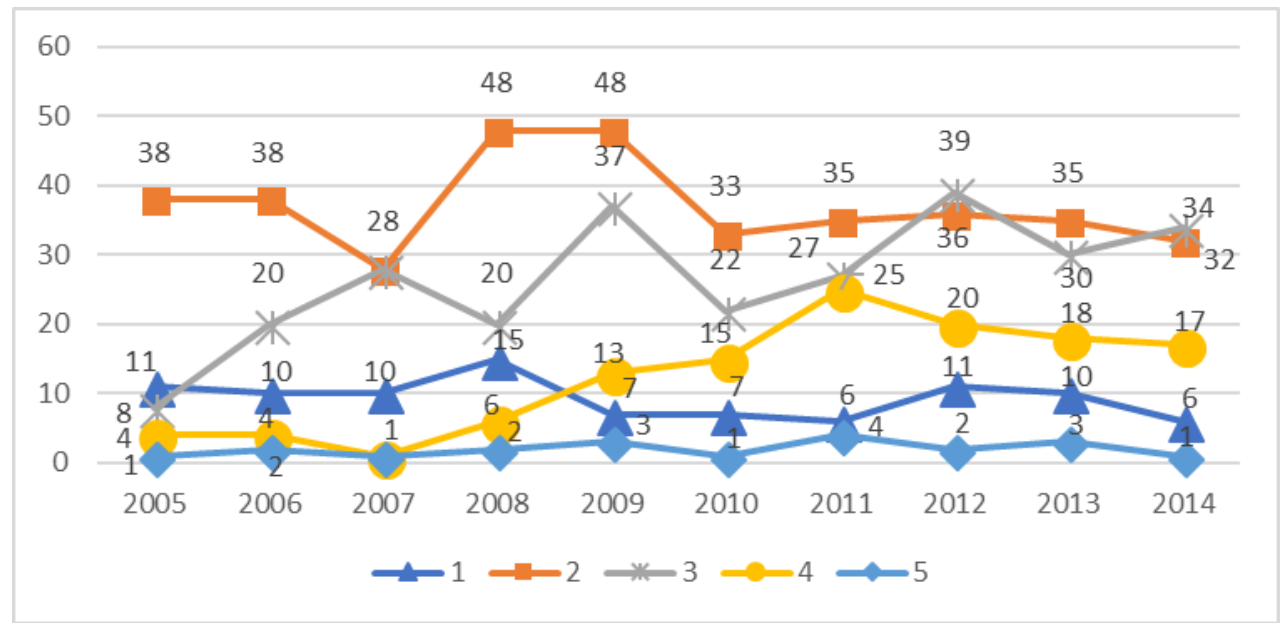

Figura 1: Evolução das coautorias de 2005 a 2014

Fonte: Elaborado pelos autores.

\subsection{Produtividade dos pesquisadores (Lotka) por contagem direta}

Pelo critério de contagem direta, cujas distribuições são apresentadas na Tabela 3, foram identificados 644 diferentes autores responsáveis pelos 873 artigos, gerando uma média de 1,36 artigos por autor e desvio-padrão de 0,93, dos quais 79,3\% publicaram apenas um artigo, portanto, uma proporção 19,3\% superior àquela sugerida por Lotka (1926), de $60 \%$, e próxima dos 78\% verificados por Leal; Almeida e Bortolon (2013) e Leal; Oliveira; Soluri (2003).

Tabela 3: Distribuição de frequência dos pesquisadores de Finanças por contagem direta

\begin{tabular}{|c|c|c|c|c|c|c|c|c|}
\hline $\begin{array}{c}\text { contribuições } \\
\text { (x) }\end{array}$ & autores (y) & $\begin{array}{c}\text { total de } \\
\text { artigos } \\
(\mathrm{x} . \mathrm{y})\end{array}$ & $\% y$ & $\log x$ & Log y & $\begin{array}{c}\text { Distribuição } \\
\text { Teórica }\end{array}$ & Diferença & $D \max$ \\
\hline 1 & 511 & 511 & 79,3 & 0,00000 & 2,70842 & 524,42 & $-13,42$ & $-0,02084$ \\
\hline 2 & 89 & 178 & 13,8 & 0,30103 & 1,94939 & 70,94 & 18,06 & 0,00721 \\
\hline 3 & 21 & 63 & 3,3 & 0,47712 & 1,32222 & 22,01 & $-1,01$ & 0,00564 \\
\hline 4 & 10 & 40 & 1,6 & 0,60206 & 1,00000 & 9,60 & 0,40 & 0,00627 \\
\hline 5 & 5 & 25 & 0,8 & 0,69897 & 0,69897 & 5,04 & $-0,04$ & 0,00621 \\
\hline 6 & 4 & 24 & 0,6 & 0,77815 & 0,60206 & 2,98 & 1,02 & 0,00779 \\
\hline 7 & 1 & 7 & 0,2 & 0,84510 & 0,00000 & 1,91 & $-0,91$ & 0,00638 \\
\hline 8 & 2 & 16 & 0,3 & 0,90309 & 0,30103 & 1,30 & 0,70 & 0,00747 \\
\hline 9 & 1 & 9 & 0,2 & 0,95424 & 0,00000 & 0,92 & 0,08 & 0,00759 \\
\hline 9 & 644 & 873 & 100 & 5,55976 & 8,58209 & 639,11 & 4,89 & 0,03372 \\
\hline$R^{2}$ & & & & & & & & \\
\hline$D_{\text {crit }}$ & & & & & & & & 0,039405 \\
\hline
\end{tabular}

Fonte: Elaborado pelos autores.

As colunas $\log ^{3}$ x e Log y foram geradas com o objetivo de se calcular o Coeficiente de Determinação de Pearson $\left(\mathrm{R}^{2}\right)^{4}$. 0 resultado do $\mathrm{R}^{2}=97,90 \%$, representa um bom ajustamento das variáveis em torno de sua reta de regressão.

A declividade dessa reta de regressão, calculada com o uso da equação (3), foi de $\mathrm{n}=-2,89$, o que representa baixa produtividade dos pesquisadores quando comparado ao estudo seminal de Lotka (1926), com parâmetro $n=-2$, e se assemelha aos resultados dos estudos nacionais anteriores em que verificou-se baixa produtividade dos pesquisadores.

\footnotetext{
3 Para esta aplicação, conforme indicado por Alvarado (2006), utilizamos o logaritmo da base 10.

40 coeficiente de correlação de Pearson, também conhecido por "coeficiente de correlação produtomomento" ou simplesmente de " $\rho$ de Pearson", é uma medida do grau da correlação (e a direção dessa correlação - se positiva ou negativa) entre duas variáveis de escala métrica.
} 
0 resultado do parâmetro $n=-2,89$, aplicado no cálculo da equação (2), retornou no parâmetro $c=0,81432$, que possibilitou gerar a distribuição teórica de Lotka com uso equação (1), cujos valores são apresentados na coluna Distribuição Teórica da Tabela 3.

Na coluna Diferença encontra-se a comparação entre as duas distribuições, empírica e teórica. Nela nota-se que as maiores diferenças ocorrem para as frequências de uma e duas contribuições, com diferenças de $-13,42$ e 18,06, respectivamente. Além disso, o total dessa coluna mostra que a distribuição empírica apresentou cinco autores a mais que o previsto.

Com a aplicação do Teste Kolmogorov-Smirnov verifica-se que o valor crítico para aceitação da igualdade das distribuições para $\mathrm{n}=644$ e nível de significância de $\alpha=0,01$, obtido pela fórmula $1,63 / \sqrt{ } n=0,039405$, quando comparado ao $\operatorname{Dmax}(0,02084<0,039405)$, remete-nos à não rejeição da hipótese de homogeneidade da distribuição de frequência empírica em relação a teórica.

Dessa forma, é possível afirmar que a distribuição da produção científica dos autores da literatura de Finanças, quando tomada pela contagem direta e quando calculada pelo Modelo do Poder Inverso Generalizado, se ajusta à Lei de Lotka, entretanto, a declividade expressa pelo parâmetro $n=-2,89$ indica que a produtividade dos autores de Finanças é inferior àquela encontrada por Lotka (1926) em seus estudos.

\subsection{Produtividade dos pesquisadores (Lotka) por contagem completa}

Pelo critério da contagem completa foram identificados 1.250 autores que contribuíram com 873 artigos dessa amostra, o que produziria uma média de 0,7 artigos por autor, entretanto, quando são consideradas as ocorrências de autoria, afinal, a maior parte dos artigos contou com os esforços de mais de um pesquisador, encontramos 2.223 ocorrências que divididas entre os 1.250 pesquisadores produz uma média de 1,77 ocorrências de um mesmo autor com desvio-padrão de 1,96 ao longo do período estudado.

Na Tabela 4 são apresentadas as colunas Log x e Log y que permitem calcular o Coeficiente de Determinação de Pearson $\left(\mathrm{R}^{2}\right)$. 0 resultado indica que o traçado do log de $\mathrm{x}$ diante do log de y em torno da reta de regressão, com $R^{2}=96,12 \%$, se mostrou elevado embora inferior ao da contagem direta.

Utilizando-se novamente a equação (3) estimou-se o valor do parâmetro $n=-2,25$, que também apontou para baixa produtividade dos pesquisadores quando comparado com a teoria, no entanto, os resultados foram melhores em relação à contagem direta, o que reflete, em certa medida, os avanços obtidos pelo exercício da colaboração em pesquisa.

Aplicando o $n=-2,25$ ao cálculo da equação (2) encontramos o parâmetro c = 0,68427 a partir do qual pode-se calcular a distribuição teórica de Lotka por meio da equação (1), cujos resultados são apresentados na coluna Distribuição Teórica, ainda na Tabela 4 .

Observando a coluna Diferença dessa tabela nota-se que algumas distribuições empíricas divergem significativamente das teóricas, principalmente nas classes de uma, três e oito contribuições, além da distribuição empírica apresentar 14 autores a mais que o previsto pela distribuição teórica. 
Tabela 4: Distribuição de frequência dos pesquisadores de Finanças por contagem completa

\begin{tabular}{|c|c|c|c|c|c|c|c|c|}
\hline $\begin{array}{c}\text { contribuições } \\
\text { (x) }\end{array}$ & autores (y) & $\begin{array}{l}\text { total de } \\
\text { artigos } \\
\text { (x.y) }\end{array}$ & $\% y$ & $\log x$ & $\log y$ & $\begin{array}{l}\text { Distribuição } \\
\text { Teórica }\end{array}$ & Diferença & $D \max$ \\
\hline 1 & 900 & 900 & 72,0 & 0,00000 & 2,95424 & 855,33 & 44,67 & 0,03573 \\
\hline 2 & 172 & 344 & 13,8 & 0,30103 & 2,23553 & 179,89 & $-7,89$ & 0,02942 \\
\hline 3 & 60 & 180 & 4,8 & 0,47712 & 1,77815 & 72,26 & $-12,26$ & 0,01962 \\
\hline 4 & 36 & 144 & 2,9 & 0,60206 & 1,55630 & 37,83 & $-1,83$ & 0,01815 \\
\hline 5 & 22 & 110 & 1,8 & 0,69897 & 1,34242 & 22,90 & $-0,90$ & 0,01743 \\
\hline 6 & 9 & 54 & 0,7 & 0,77815 & 0,95424 & 15,20 & $-6,20$ & 0,01247 \\
\hline 7 & 10 & 70 & 0,8 & 0,84510 & 1,00000 & 10,74 & $-0,74$ & 0,01188 \\
\hline 8 & 16 & 128 & 1,3 & 0,90309 & 1,20412 & 7,96 & 8,04 & 0,01831 \\
\hline 9 & 8 & 72 & 0,6 & 0,95424 & 0,90309 & 6,10 & 1,90 & 0,01983 \\
\hline 10 & 2 & 20 & 0,2 & 1,00000 & 0,30103 & 4,82 & $-2,82$ & 0,01758 \\
\hline 11 & 2 & 22 & 0,2 & 1,04139 & 0,30103 & 3,89 & $-1,89$ & 0,01607 \\
\hline 12 & 6 & 72 & 0,5 & 1,07918 & 0,77815 & 3,20 & 2,80 & 0,01831 \\
\hline 13 & 2 & 26 & 0,2 & 1,11394 & 0,30103 & 2,67 & $-0,67$ & 0,01777 \\
\hline 14 & 2 & 28 & 0,2 & 1,14613 & 0,30103 & 2,26 & $-0,26$ & 0,01757 \\
\hline 15 & 2 & 30 & 0,2 & 1,17609 & 0,30103 & 1,93 & 0,07 & 0,01762 \\
\hline 16 & 0 & 0 & 0,0 & 1,20412 & 0,00000 & 1,67 & $-1,67$ & 0,01628 \\
\hline 17 & 0 & 0 & 0,0 & 1,23045 & 0,00000 & 1,46 & $-1,46$ & 0,01511 \\
\hline 18 & 0 & 0 & 0,0 & 1,25527 & 0,00000 & 1,28 & $-1,28$ & 0,01408 \\
\hline 19 & 0 & 0 & 0,0 & 1,27875 & 0,00000 & 1,14 & $-1,14$ & 0,01317 \\
\hline 20 & 0 & 0 & 0,0 & 1,30103 & 0,00000 & 1,01 & $-1,01$ & 0,01236 \\
\hline 21 & 0 & 0 & 0,0 & 1,32222 & 0,00000 & 0,91 & $-0,91$ & 0,01164 \\
\hline 22 & 0 & 0 & 0,0 & 1,34242 & 0,00000 & 0,82 & $-0,82$ & 0,01098 \\
\hline 23 & 1 & 23 & 0,1 & 1,36173 & 0,00000 & 0,74 & 0,26 & 0,01119 \\
\hline Totais & 1250 & 2223 & 100,0 & 22,41249 & 16,2114 & 1236,01 & 13,99 & 0,39258 \\
\hline$R^{2}$ & \multirow{2}{*}{\multicolumn{7}{|c|}{96,12}} & \\
\hline$D_{\text {crit }}$ & & & & & & & & 0,02828 \\
\hline
\end{tabular}

Fonte: Elaborado pelos autores.

Alvarado (2006) menciona que há uma corrente de pesquisadores que desenvolvem estudos bibliométricos que defendem a possibilidade de se retirar pares de dados para encontrar a melhor distribuição dos pontos em torno da reta, contudo, segue-se neste trabalho a corrente alternativa que sugere que os dados não devam ser retirados e sim tratados na sua forma bruta.

No Teste Kolmogorov-Smirnov , o valor crítico (D_crit) encontrado para aceitação da igualdade das distribuições, para uma amostra $\operatorname{com} n=1.250$ e nível de significância de $\alpha=$ 0,01 , foi de $D_{-}$crit $=0,02828$ que, quando comparando ao $\operatorname{Dmax}=0,03573$, remete-nos à rejeição da hipótese de homogeneidade da distribuição de frequência empírica em relação à teórica.

Essa constatação permite afirmar que a distribuição da produção científica dos autores da literatura de Finanças, quando tomada pela contagem completa e calculada pelo Modelo do Poder Inverso Generalizado, não se ajusta à Lei de Lotka.

Quando comparamos o coeficiente de inclinação da contagem direta $(n=-2,89)$ com o da contagem completa $(n=-2,25)$ notamos que a produção em regime de colaboração proporcionou melhor resultado, porém, o elevado número de pesquisadores com apenas uma publicação ainda demonstra ser uma barreira a ser superada pela área de Finanças, tal como já foi apontado em trabalhos anteriores.

\section{CONSIDERAÇÕES FINAIS}

A presente pesquisa analisou o comportamento da produção científica da área de Finanças, a partir de uma base de dados composta por 873 artigos, colhidos em 24 conceituados periódicos nacionais da área de Administração, Ciências Contábeis e Turismo, classificados nos estratos Qualis/CAPES A2, B1 e B2 do ano de 2014, num período de cobertura que começa em 2005 e se estende até 2014.

Verificou-se que a produção científica em Finanças vem seguindo uma tendência de crescimento de, em média, 5,97\% ao ano, porém, apresentando um comportamento cíclico, provavelmente influenciado pelos antigos ciclos trienais, hoje quadrienais, de avaliação dos Programas de Pós-Graduação Stricto Sensu, cujos primeiros anos coincidem com os de baixa produção e os últimos com os de alta. 
As coautorias foram formadas, em sua maioria, por dois $(42,5 \%)$ pesquisadores, seguida pelas de três $(30,36 \%)$ e quatro $(14,09 \%)$ pesquisadores, sendo que a formação com dois pesquisadores foi a mais frequente de 2005 até 2011, superada pela formação de três pesquisadores em 2012 e 2014 a qual demonstra ser a nova tendência na formação de coautorias.

Nos testes de adequação da distribuição de frequência empírica àquela proposta pela Lei de Lotka, utilizando-se do método de contagem direta, confirmou-se a hipótese de homogeneidade da distribuição permitindo afirmar que a distribuição da produção científica dos autores da literatura de Finanças se ajusta à Lei de Lotka, contudo, a declividade expressa pelo parâmetro $n=-2,89$ indicou que a produtividade dos autores de Finanças é inferior àquela encontrada por Lotka (1926) em seus estudos.

Utilizando o método de contagem completa, os resultados remetem à rejeição da hipótese de homogeneidade das distribuições levando-nos a afirmar que a distribuição da produção científica dos autores da literatura de Finanças não se ajusta à Lei de Lotka. Além disso, a declividade expressa pelo parâmetro $n=-2,25$ indica que a produtividade dos autores de Finanças também foi inferior àquela encontrada por Lotka (1926), porém, superior à encontrada pela contagem direta, indicando um efeito favorável da produção em regime de colaboração.

Cabe destacar que, em ambas as formas de contagem, direta e completa, o elevado número de pesquisadores com apenas uma publicação, 79,3\% e 72\% respectivamente, foram superiores aos $60 \%$ previstos por Lotka, influenciando negativamente a produtividade dos pesquisadores e, ao que parece, estaria associado às exigências protocolares de publicação dos trabalhos de conclusão de mestrado e doutorado, como já foi apontado por Camargos, Silva e Dias (2009) e Leal, Almeida e Bortolon (2013).

Como limitações do presente estudo, podemos apontar o enfoque fundamentalmente quantitativo, o fato de contemplar apenas periódicos nacionais, além da subjetividade intrínseca nas fases de seleção dos artigos, contudo, grande parte das decisões foram tomadas com o objetivo de ampliar a amostra, e não o contrário.

Por fim, sugere-se que sejam realizados novos estudos aplicando técnicas de classificação textual automatizadas, que diminuam a subjetividade no processo de classificação e que contribuam para uma melhor compreensão das contribuições de pesquisadores e periódicos em nível temático além da utilização técnicas qualitativas que permitam aprofundamento sobre as reais dificuldades vivenciadas pelos pesquisadores na produção e publicação de seus trabalhos.

\section{AGRADECIMENTOS}

Agradecimentos especiais à Comissão de Aperfeiçoamento de Pessoal de Ensino Superior (CAPES), pela Bolsa CAPES/PROSUP concedida ao autor; à Fundação de Amparo à Pesquisa do Estado de São Paulo (FAPESP), processo 15/25627-1, pelo apoio financeiro para apresentação do trabalho no EnANPAD 2017 e à SPHINX BRASIL pela concessão da licença estudante do SPHINX Léxica.

\section{REFERÊNCIAS}

ALVARADO, R. U. A produtividade dos autores na literatura de enfermagem um modelo de aplicação da lei de Lotka. Informação \& Sociedade: Estudos, v. 16, n. 1, p. 63-78, 2006. Disponível em: < http://www.brapci.inf.br/_repositorio/2010/11/pdf_d9d46123dc_0012843.pdf>

ALVARADO, R. U. A Lei de Lotka e a Produtividade dos Autores. Belo Horizonte: Universidade Federal de Minas Gerais, 2007.

ARAÚJO, C. A. Bibliometria : evolução histórica e questões atuais. Em Questão, v. 12, n. 1, p. 11-32, 2006. Disponível em: <http://seer.ufrgs.br/index.php/EmQuestao/article/view/16>

BOROKHOVICH, K. A.; BRICKER, R. J.; SIMKINS, B. J. Financial Management's Sucess as an Academic Journal. Financial Management, v. 28, n. 3, p. 76-82, 1999. 
CAMARGOS, M. A. DE; COUTINHO, E. S.; AMARAL, H. F. o Perfil da Área de Finanças do EnANPAD: Um Levantamento da Produção Científica e de suas Tendências entre 20002004. In: ENCONTRO DA ASSOCIAÇÃO NACIONAL DE PÓS GRADUAÇÃO E PESQUISA EM ADMINISTRAÇÃO, 30, Salvador, 2006. Anais...2006

CAMARGOS, M.; SILVA, W.; DIAS, A. Análise da produção científica em finanças entre 2000-2008: um estudo bibliométrico dos encontros da ANPAD. In: ENCONTRO DA ASSOCIAÇÃO NACIONAL DE PÓS GRADUAÇÃO E PESQUISA EM ADMINISTRAÇÃO, 33, São Paulo. Anais...2009

COSTA-NETO, P. L. DE O. Estatística. 1. ed. São Paulo: Edgard Blücher, 1977.

COX, R. A K.; CHUNG, K. H. Patterns of Productivity in the Finance Literature: A Study of the Bibliometric Distributions. The Journal of Finance, v. 45, n. 1, p. 301-309, 1990.

DRESDEN, A. A report on the scientific work of the Chicago section. Bulletin of the American Mathematical Society, v. 28, n. 6, p. 303-307, 1922.

FONSECA, E. N. Bibliometria: Toeria e Prática. 1. ed. São Paulo: Cultrix: Editora da Universidade de São Paulo, 1986.

GIL, A. C. Como elaborar projetos de pesquisa. 5. ed. São Paulo: Atlas, 2010.

GUEDES, L. S. G.; BORSCHIVER, S. Bibliometria: uma ferramenta estatística para a gestão da informação e do conhecimento, em sistemas de informação, de comunicação e de avaliação científica e tecnológica. In: ENCONTRO NACIONAL DE CIÊNCIA DA INFORMAÇÃO, 6, Salvador. Anais...2005. Disponível em: <http://www.cinformanteriores.ufba.br/vi_anais/>.

HECK, J. L.; COOLEY, P. L. Prolific Authors in the Finance Literature : A Half Century of Contributions. Journal of Finance Literature, v. 1, n. 1992, p. 1-23, 2002.

HECK, J. L.; COOLEY, P. L.; HUBBARD, C. M. Contributing Authors and Institutions to the Journal of Finance: 1946-1985. Journal of Finance, v. 41, n. 5, p. 1129-1140, 1986.

HERLING, L. H. D.; LIMA, M. V. A. DE; MORITZ, G. D. O. Finanças corporativas: sua organização e base epistemológica. In: COLÓQUIO INTERNACIONAL DE EPISTEMOLOGIA E SOCIOLOGIA DA CIÊNCIA DA ADMINISTRAÇÃO, 3, Fllorianópolis. Anais...2013. Disponível em: <http://www.coloquioepistemologia.com.br/anais2013/ANE113.pdf>

LEAL, R. P. C.; OLIVEIRA, J. DE; SOLURI, A. F. Perfil da pesquisa em finanças no Brasil. Revista de Administração de Empresas, São Paulo, v.43, n.1, p. 91-104, 2003. Disponível em: <http://www.scielo.br/scielo.php?script=sci_arttext\&pid=S0034-75902003000100010>

SPHINX Brasil. LÉXICA: Sistema para pesquisa e análise de dados. 2000. Disponível em: $<$ http://www.sphinxbrasil.com>.

LOTKA, A. J. The freq distrib of scientific productivity. Journal of the Washington Academy of Sciences, v. 16, n. 12, p. 317-323, 1926.

MATTOS, P. L. C. L. DE. "Bibliometria": a metodologia acadêmica convencional em questão. RAE eletrônica, v. 3, n. 2, p. 1-6, 2004. Disponíel em:

<http://www.scielo.br/pdf/raeel/v3n2/v3n2a16>.

PAO, M. L. Lotka's Law: a testing procedure. Processing and Management, v. 21, n. 4, p. 305-320, 1985.

POTTER, W. G. Lotka's Law Revisited. Library Resources \& Technical Services, v. 30, n. 1, p. 21-40, 1981.

RICHARDSON, R. J. Pesquisa Social: Métodos e Técnicas. 3. ed. São Paulo: Atlas, 2015.

SIEGEL, S. Estatística não-paramétrica para as ciências do comportamento. $1^{\mathrm{a}}$ ed. São Paulo: McGraw-Hill, 1975.

SPLITTER, K.; DA ROSA, C. A.; BORBA, J. A. Uma Análise das Características dos Trabalhos "Ditos" Bibliométricos Publicados no EnANPAD entre 2000 e 2011EnANPAD. In: 
ENCONTRO DA ASSOCIAÇÃO NACIONAL DE PÓS GRADUAÇÃO E PESQUISA EM ADMINISTRAÇÃO, 36, Rio de Janeiro, 2012. Rio de Janeiro: ANPAD, 2012.

TEIXEIRA, M. L. M.; IWAMOTO, H. M.; MEDEIROS, A. L. Estudos bibliométricos (?) em administração: discutindo a transposição de finalidade. Administração: Ensino e Pesquisa, v. 14, n. 3, p. 423-452, 2013.

VASCONCELOS, M. P. B. et al. Produção científica brasileira em finanças no período 20002010. RAE- Revista de Administração de Empresas, v. 53, n. 1, p. 46-55, 2013.

VERGARA, S. C. Projetos e Relatórios de Pesquisa em Administração. 15. ed. São Paulo: Atlas, 2014.

WESTON, J. F. Finanças de Empresas: campo e metologia. 1ª ed. São Paulo: Atlas, 1977.

Editores do artigo: Enrique Muriel-Torrado, Edgar Bisset Alvarez, Camila Barros. 